\title{
BIOMOLECULES MEDIATED TARGETING OF VASCULAR ENDOTHELIAL GROWTH FACTOR IN NEURONAL DYSFUNCTION: AN IN SILICO APPROACH
}

\author{
NIRAJ KUMAR JHA ${ }^{1}$, PRAVIR KUMAR ${ }^{2 *}$ \\ ${ }^{1}$ Department of Biotechnology, Molecular Neuroscience and Functional Genomics Laboratory, Delhi Technological University (Formerly \\ DCE), New Delhi - 110 042, India. ${ }^{2}$ Department of Biotechnology, Delhi Technological University (Formerly Delhi College of Engineering), \\ New Delhi - 110 042, India. Email: pravirkumar@dce.edu \\ Received: 01 May 2017, Revised and Accepted: 20 May 2017
}

ABSTRACT

Objective: Neurodegenerative diseases are a debilitating age-related disorder manifested by memory loss, impaired motor activity, and loss of muscle tone due to the accumulation of toxic metabolites in the brain. Despite the knowledge of factors causing neurodegenerative disorders, it remains irreversible and incurable. Growing evidence have currently advocated the physiological and pathological contribution of hypoxia-induced vascular endothelial growth factor (VEGF) in neuronal loss. The objective of this research report highlights biomolecules mediated targeting of VEGF activity based on in silico approaches that could establish a potential therapeutic window for the treatment of different abnormalities associated with impaired VEGF.

Methods: We employed various in silico methods such as drug-likeness parameters, namely, Lipinski filter analysis, Pock Drug tool for active site prediction, AUTODOCK 4.2.1, and LigPlot1.4.5 for molecular docking studies.

Results: Three-dimensional structure of VEGF was generated and Ramachandran plot obtained for quality assessment. RAMPAGE displayed $99.5 \%$ of residues in the most favored regions, $0.5 \%$ residues in additionally allowed, and no residues in disallowed regions in VEGF, showing that stereochemical quality of protein structure is good. Further, initial screenings of the molecules were done based on Lipinski's rule of five. Finally, we have found Naringenin to be most effective among three biomolecules in modulating VEGF activity based on minimum inhibition constant, Ki, and highest negative free energy of binding with the maximum interacting surface area during docking studies.

Conclusion: The present study outlines the novel potential of biomolecules in regulating VEGF activity for the treatment of different abnormalities associated with impaired VEGF

Keywords: Hypoxia, Vascular endothelial growth factor, Biomolecules, Active site prediction, Molecular docking

(C) 2017 The Authors. Published by Innovare Academic Sciences Pvt Ltd. This is an open access article under the CC BY license (http://creativecommons. org/licenses/by/4. 0/) DOI: http://dx.doi.org/10.22159/ajpcr.2017.v10i9.19466

\section{INTRODUCTION}

Neurodegenerative diseases are pathological conditions that have an insidious onset and chronic progression. Different models have been established to study these diseases to understand their underlying mechanisms and to investigate new therapeutic strategies [1]. Several downstream signaling molecules are reported to trigger under hypoxia. Vascular endothelial growth factor (VEGF) is one of them, which is responsible for the formation of new blood vessels (angiogenesis) and lead to the supply of nutrients and oxygen for normal homeostasis [2]. Moreover, the crucial role of VEGF in the brain is not restricted only to controlling vessel growth: But it has direct effects on different types of neural cells including neural stem cells. Conversely, altered expression of this molecule has been implicated in virtually every type of angiogenic disorder, including those associated with cancer, ischemia, and inflammation [3]. Moreover, studies have also revealed the pathological implication of VEGF in the progression of neurodegenerative disorders (NDDs) including, Alzheimer's disease, Huntington disease, and amyotrophic lateral sclerosis. Recent genetic studies have revealed that reduced VEGF levels cause neurodegeneneration through impairing neural tissue perfusion [4]. Importantly, implementation of different biomolecules may helpful in regulating the altered levels of VEGF in cells. The growing evidence for an etiologic role of VEGF in neurodegeneration provides an underlying principle for considering the therapeutic potential of VEGF for NDDs, which are mostly not curable. In this framework, we have introduced different biomolecules (naringenin, quercetin, and sesamol) for targeting VEGF. These biomolecules have chemoprotective, anti- inflammatory, neuroprotective, and anti-aging property. For this purpose, we have performed in silico based structural and functional analysis of these molecules for revealing its therapeutic importance against neuronal loss through modulating the impaired expression of VEGF. The objective of this study is to explore neuroprotective action of these biomolecules in regulating the altered level of VEGF to attenuate the toxicity associated with toxic proteins in neuronal death.

\section{METHODS}

Visualization and quality assessment of three-dimensional (3D)structure of VEGF

3D-structure of VEGF (ID: 1QTY) was generated using protein data bank (PDB), structural evaluation, and stereochemical analysis was performed using RAMPAGE (http://www.mordred.bioc.cam. ac.uk/ rapper/rampage.php). Errat server was used to find the accuracy of the structure and visualization of determined structures was performed using University of California, San Francisco Chimera.

\section{Active site prediction}

The active sites of VEGF were predicted using the Pock Drug tool (http:// pockdrug.rpbs.univ-paris-diderot.fr/cgi-bin/index.py?page=home). The PDB structure of VEGF was uploaded and active sites were predicted using f-pocket estimation and setting ligand proximity threshold at 5.5.

\section{Ligand optimization}

Reported ligand molecules along with their physical and chemical properties were retrieved from PubChem Compound Database (http:// 
www.pubchem.ncbi.nlm.nih.gov/). PubChem is a composite database that is backed up by three primary databases, i.e., polycarbonate compounds (PC) substance, PC compund, and PC BioAssay. PubChem provides biological activity and chemical information of small molecules. PC substance contains information about the substances; PC compound contains information about chemical compounds, and PC BioAssay provides information about Bioassays. Three compounds (naringenin, quercetin, and sesamol) were selected. SDF files of ligands were converted in PDB file with the help of Open Babel tool that could be used for docking study. Visualization of molecular structure of compounds was done using PyMOL.

\section{Lipinski filter analysis of screened drugs}

An online tool Lipinski filter (http://www.scfbio-iitd.res.in/software/ drugdesign/lipinski.jsp) was used to retrieve the information about drug-likeness properties of biomolecules with the help of Lipinski rule of five. Lipinski rule helps differentiate drug and non-drug such as properties of molecules. It is used to identify the possibility of success or failure due to drug-likeness for molecules fulfiling with two or more of the following rules: (a) Molecular mass should be $<500 \mathrm{Da}$, (b) high lipophilicity (expressed as $\log \mathrm{p}<5$ ), (c) $<5$ hydrogen bond donors, (d) $<10$ hydrogen bond acceptors, and (e) molar refractivity should be between 40 and 130 .

\section{Preparation of protein and ligand molecules}

Preparation of protein involves the addition of polar hydrogen atoms, neutralization of charge, and removal of any miscellaneous structures from the protein molecule by AUTODOCK 4.2.1 whereas ligand preparation involves the neutralization of charge.

\section{Molecular docking studies}

Prepared and optimized structures of ligands and protein were ultimately used for molecular docking using AUTODOCK 4.2.1 for predicting the possible protein-ligand interactions and the results that include the understanding of the association that involves H-bonding, and hydrophobic interactions were analyzed using LIGPLOT1.4.5, a program to generate schematic diagrams of protein-ligand interactions.

\section{RESULTS}

\section{D-structure visualization and quality assessment}

3D-structure of VEGF was generated and visualized using UCSF Chimera (Fig. 1a). Even though, there were no steric clashes in the structure generated, it was assessed for geometric and energy aspects. Ramachandran plot was used to check the reliability of predicted 3D-structure of VEGF. RAMPAGE checks the stereochemical quality of a protein structure by analyzing residue-by-residue geometry and overall structural geometry. Ramachandran plot was obtained for VEGF for quality assessment. RAMPAGE displayed $99.5 \%$ of residues in the most favored regions, $0.5 \%$ residues in additionally allowed, and no residues in disallowed regions in VEGF (Fig. 1b). Errat server was used to determine the accuracy of the model. Result of Errat showed 95.694\% accurate structure for VEGF

\section{Active site prediction}

Of top 10 pockets, VEGF had best pocket at $\mathrm{P} 4$ with a drug ability score of 0.95 and 0.01 standard deviation (Table 1). The volume of given pocket was 1338.57 cubic angstroms and fourteen residues were involved in interaction at this site.

\section{Lipinski filter analysis of screened drugs}

Further, the screening of ligand molecules was done on the basis of Lipinski's rule of five. Lipinski filter analysis revealed that all the compounds selected possessed drug likeness and can be used for docking purposes (Fig. 2).

\section{Molecular docking of VEGF with biomolecules}

Biomolecules bound to VEGF at P4 pocket and same residues as predicted were involved in the interaction. The estimated free energy of binding for VEGF and naringenin was - $7.56 \mathrm{kcal} / \mathrm{mol}$ and total intermolecular energy was $-9.05 \mathrm{kcal} / \mathrm{mol}$. Similarly, the estimated free energy of binding for VEGF and quercetin was $-7.10 \mathrm{kcal} / \mathrm{mol}$ and total intermolecular energy was $-8.89 \mathrm{kcal} / \mathrm{mol}$. Likewise, the estimated free energy of binding for VEGF and sesamol was - $5.09 \mathrm{kcal} / \mathrm{mol}$ and total intermolecular energy was $-5.39 \mathrm{kcal} / \mathrm{mol}$. Molecular docking pattern of VEGF with screened molecules (naringenin, quercetin, and sesamol) have been identified and depicted in Fig. 3. On the basis of docking analysis, interacting compounds with minimum binding constant and highest negative free energy of binding are most effective. Docking calculation of VEGF with these molecules has been presented in Table 2 .

Binding site of VEGF with selected compounds along with its reported Inhibitory active site

Binding site residues of VEGF interacting with naringenin, quercetin, and sesamol were found to be the same as the residues involved in their respective catalytic sites. Interacting residues of VEGF with naringenin, quercetin, and sesamol along with their identified catalytic sites have
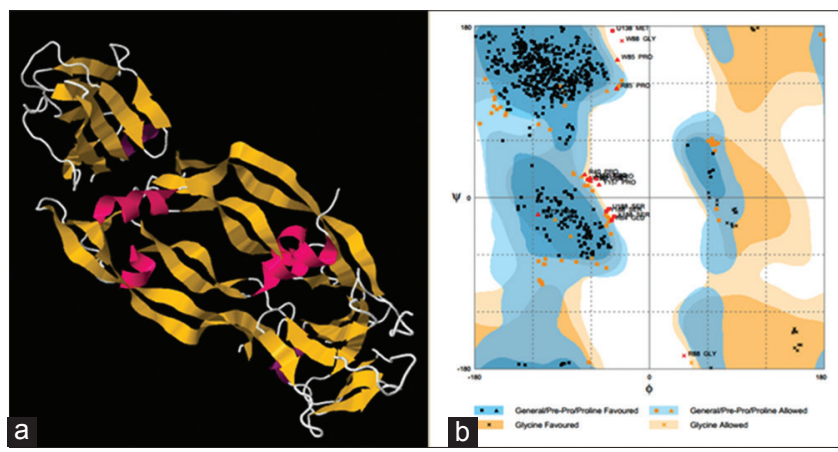

Fig. 1: (a) Three-dimensional-structure and (b) Ramachandran plot of vascular endothelial growth factor protein

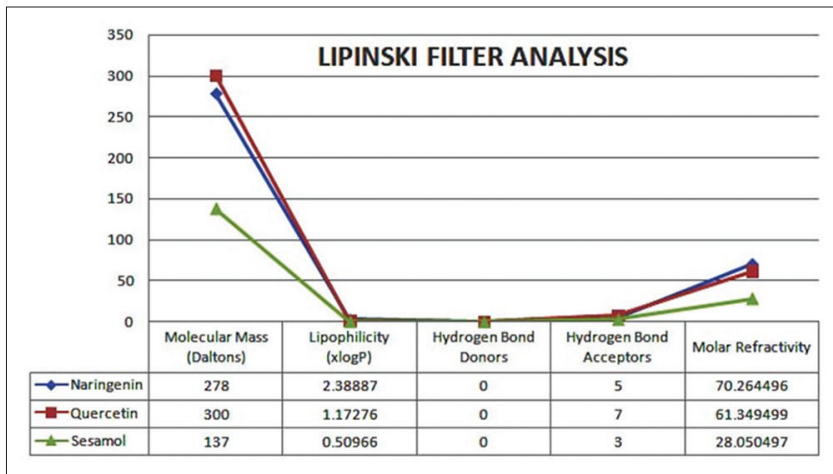

Fig. 2: Differentiation of drugs on the basis of Lipinski rule of five by Lipinski filter

Table 1: Active sites of vascular endothelial growth factor

\begin{tabular}{|c|c|c|c|c|c|c|c|c|}
\hline Pockets ${ }^{4}$ & $\begin{array}{l}\text { Vol. } \\
\text { Hull }\end{array}$ & $\begin{array}{l}\text { Hydroph. } \\
\text { Kyte* }\end{array}$ & $\begin{array}{l}\text { Polar } \\
\text { Res: }\end{array}$ & $\begin{array}{l}\text { Aromatic } \\
\text { Res.: }\end{array}$ & $\begin{array}{l}\text { otyr } \\
\text { atom }\end{array}$ & $\begin{array}{l}\text { Nb. } \\
\text { Res. }\end{array}$ & $\begin{array}{l}\text { Drugg } \\
\text { Prob: }\end{array}$ & $\begin{array}{l}\text { Standard } \\
\text { Doviation }\end{array}$ \\
\hline PO & 2205.45 & .0 .58 & 0.67 & 0.2 & 0.0 & 30.0 & 0.63 & 0.09 \\
\hline P1 & 2500.5 & .0 .67 & 0.66 & 0.19 & 0.0 & 32.0 & 0.59 & 0.08 \\
\hline P 16 & 452.91 & 0.83 & 0.43 & 0.0 & 0.0 & 14.0 & 0.93 & 0.02 \\
\hline P 18 & 744.97 & 0.56 & 0.5 & 0.14 & 0.0 & 14.0 & 0.94 & 0.01 \\
\hline P 19 & 455.66 & 0.83 & 0.43 & 0.0 & 0.0 & 14.0 & 0.93 & 0.02 \\
\hline $\mathbf{P} 2$ & 1622.2 & -0.86 & 0.68 & 0.24 & 0.0 & 25.0 & 0.49 & 0.12 \\
\hline P3 & 1819.24 & -0.87 & 0.69 & 0.21 & 0.03 & 29.0 & 0.53 & 0.03 \\
\hline P 4 & 1338.57 & 0.34 & 0.62 & 0.19 & 0.04 & 21.0 & 0.95 & 0.01 \\
\hline P 5 & 1159.82 & -0.3 & 0.67 & 0.24 & 0.04 & 21.0 & 0.83 & 0.02 \\
\hline P 6 & 1368.17 & -1.2 & 0.75 & 0.06 & 0.0 & 16.0 & 0.14 & 0.02 \\
\hline
\end{tabular}


been show in Table 3 and their two-dimensional and 3D pattern of interaction is presented in Fig. 4.

\section{DISCUSSION}

Neurodegeneration is an umbrella term for a range of conditions which primarily affect the neurons in the human brain [5]. Despite the knowledge of various factors which contribute in the occurrence and progression of NDDs, the exact cause and cure remains elusive. Abnormal expression of VEGF protein in terminally differentiated neurons is a recently known phenomenon which has been shown to drive neurodegeneration followed by apoptosis [6]. Free-radical injury of microvessels under hypoxia causes neuroinflammation and oligemia which thereafter leads to $A \beta$ accumulation through vascular damage and the activation of proangiogenic factors including, hypoxiainducible factor- $1 \alpha$, and VEGF-1 [7]. Further, invading macrophages and monocytes also causes neuronal damage through activation of VEGF-1 [8]. Similarly, low VEGF levels not only impair spinal cord perfusion and cause chronic ischemia of motoneurons but also deprive these cells of vital VEGF-dependent survival and neuroprotective signals.

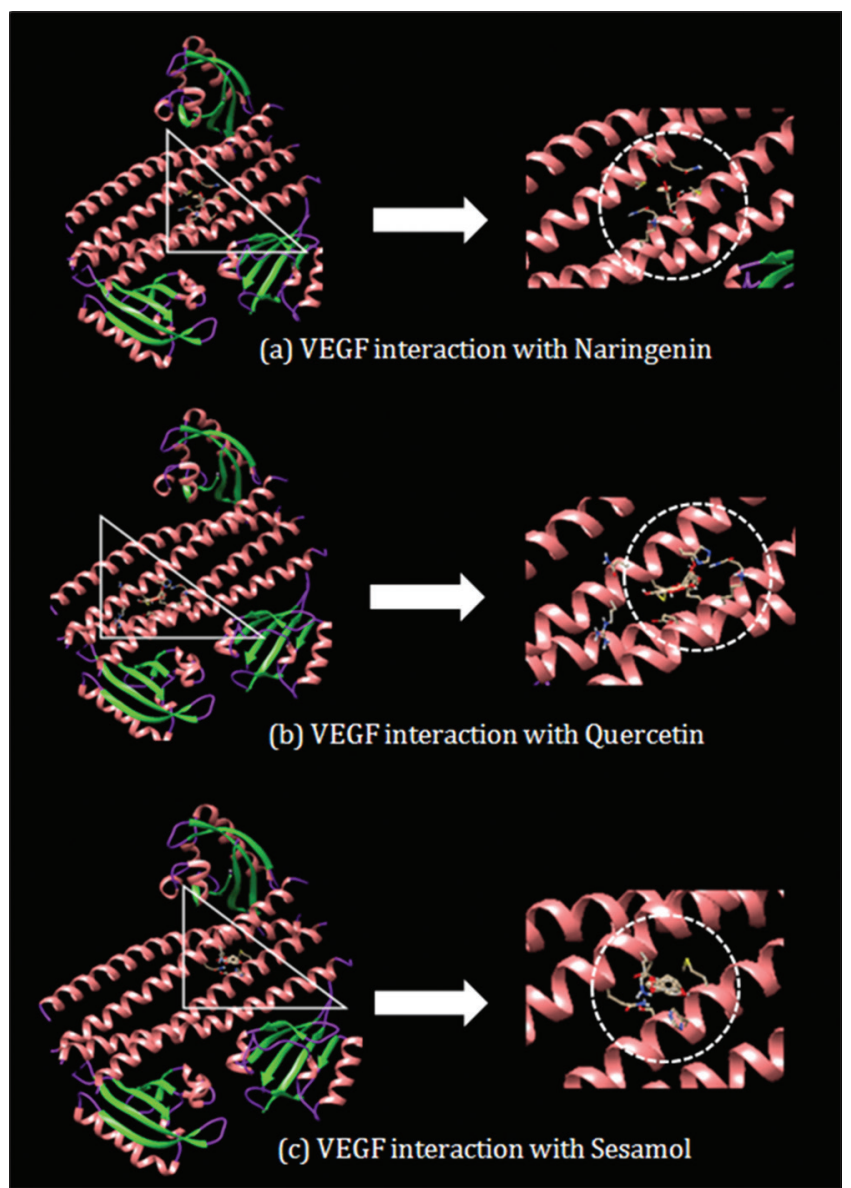

Fig. 3: Binding of vascular endothelial growth factor with selected compounds
Both phenomenons result in progressive degeneration of motoneurons, associated with muscle weakness, paralysis, and death [9]. Thus, it seems imperative to design therapeutic strategies aimed at attenuating the altered level of VEGF to inhibit the cascade of neurodegeneration.

Flavonoids have been advocated to exert human health benefits by antioxidant and anti-inflammatory mechanisms [10]. Naringenin reportedly prevent oxidative stress and nuclear factor kappa B (NF- $\mathrm{B}$ )mediated inflammatory brain damage in the rat model of focal cerebral injury. Further, prophylactic treatment with naringenin ameliorated functional outcomes and abrogated the ischemic brain injury by

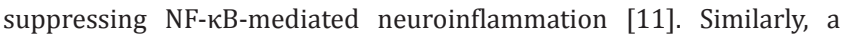
significant raise in neuronal survivability was observed with quercetin treatment in rats administered 6-OHDA. Both naringenin and quercetin also reversed the effect of hypobaric hypoxia and elicit neuroprotective response by reducing VEGF level in the murine model [12]. Further, sesamol pre-treatment restored oxidative defense possibly by its free radical scavenging lighted the neuroprotective effect of sesamol against 3-NP-induced neuronal damage [13]. Taken together, all these data provide convincing evidence of using VEGF interaction bioflavonoids such as naringenin and quercetin in attenuating the level of VEGF and in turn, inhibit the cascade of neuronal death.

RAMPAGE displayed $99.5 \%$ of residues in the most favored regions, $0.5 \%$ residues in additionally allowed and no residues in disallowed regions in VEGF, showing that stereochemical quality of protein structure is good. Result of Errat showed $95.694 \%$ accurate structure for VEGF. Lipinski filter analysis of all the compounds revealed that these compounds could act such as a drug and have drug-like property as these compounds meet the criteria of Lipinski Rule of five. Finally, molecular docking studies indicated that all these compounds can bind to and modulate the level of VEGF and possibly, halt or inhibit toxic proteins induced neuronal death in NDDs. Docking study revealed that all three compounds are interacting at the reported active binding site and binding atomic coordination was compared with the template complex coordination and found that docked drug coordination was similar with the known coordination. Amino acid residues of VEGF involved in interaction with naringenin, quercetin, and sesamol were found to be the same as the residues involved in binding with earlier used inhibitors. These observations clearly indicate that we can efficiently determine active site coordinates to investigate the effect of inhibitors on the functional active site of protein. In this result, the most effective compound was found to be naringenin as showing minimum inhibition constant, $\mathrm{Ki}$, and lowest free energy of binding with maximum interacting surface area [14-17]. These findings can be further validated through in vitro and in vivo studies in neurodegeneration. Overall, although further work is required, these studies advocate the pivotal role of VEGF in NDDs and provide adequate grounds for estimating the potential therapeutic effectiveness of VEGF in their management.

\section{CONCLUSION}

The results of our study provide novel potential of biomolecules such as naringenin, quercetin, and sesamol in regulating VEGF expression in the brain, which has wider implications in the progression as well as protection against NDDs. Moreover, of these three biomolecules naringenin is showing better interaction with VEGF based on their minimum binding constant and highest negative free energy.

Table 2: Docking calculation of compounds with VEGF

\begin{tabular}{|c|c|c|c|c|c|c|c|}
\hline $\begin{array}{l}\text { Compound } \\
\text { name }\end{array}$ & $\begin{array}{l}\text { Estimated } \\
\text { free energy } \\
\text { of binding } \\
\text { (kcal/mol) }\end{array}$ & $\begin{array}{l}\text { Estimated } \\
\text { binding } \\
\text { constant }(\mu M)\end{array}$ & $\begin{array}{l}\text { Estimated } \\
\text { intermolecular } \\
\text { energy } \\
\text { (kcal/mol) }\end{array}$ & $\begin{array}{l}\text { vdW+Hbond+desolv } \\
\text { energy (kcal/mol) }\end{array}$ & $\begin{array}{l}\text { Electrostatic } \\
\text { energy } \\
\text { (kcal/mol) }\end{array}$ & $\begin{array}{l}\text { Estimated } \\
\text { internal } \\
\text { energy } \\
\text { (kcal/mol) }\end{array}$ & $\begin{array}{l}\text { Torsional } \\
\text { free energy } \\
\text { (kcal/mol) }\end{array}$ \\
\hline Naringenin & -7.56 & 1.74 & -9.05 & -9.03 & -0.02 & +9.69 & +1.19 \\
\hline Quercetin & -7.10 & 6.21 & -8.89 & -8.73 & -0.16 & +9.49 & +1.79 \\
\hline Sesamol & -5.09 & 186.16 & -5.39 & -5.33 & -0.06 & +0.33 & +0.30 \\
\hline
\end{tabular}

VEGF: Vascular endothelial growth factor 
Table 3: VEGF known inhibitory site and selected compounds interacting residues

\begin{tabular}{|c|c|}
\hline Compounds & Interacting residues \\
\hline Reported active site & 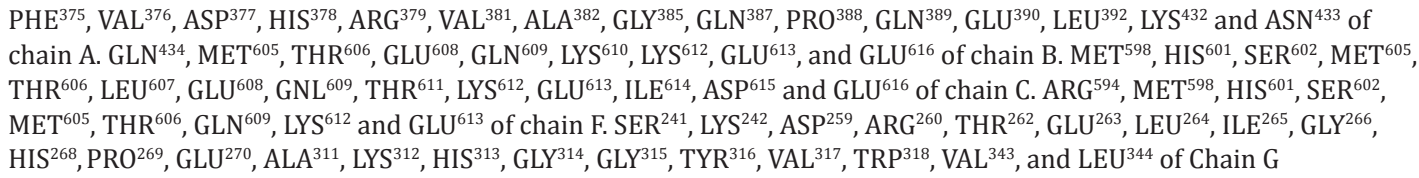 \\
\hline Naringenin & $\mathrm{MET}^{605}, \mathrm{THR}^{606}, \mathrm{GLU}^{608}$, and GLN ${ }^{609}$ residues of chain C and MET 605 , THR ${ }^{606}$, and $\mathrm{GLN}^{609}$ residues of chain $\mathrm{F}$ \\
\hline Quercetin & $\mathrm{GLN}^{609}, \mathrm{LYS}^{612}, \mathrm{GLU}^{613}$, and $\mathrm{GLU}^{616}$ residues of chain $\mathrm{C}$ and $\mathrm{ARG}^{594}, \mathrm{MET}^{598}, \mathrm{HIS}^{601}$, and $\mathrm{SER}^{602}$ residues of chain $\mathrm{F}$ \\
\hline Sesamol & $\mathrm{MET}^{598}, \mathrm{HIS}^{601}$, and $\mathrm{SER}^{602}$ residues of chain $\mathrm{C}$ and $\mathrm{GLN}^{609}, \mathrm{LYS}^{612}$, and $\mathrm{GLU}^{613}$ residues of chain $\mathrm{F}$ \\
\hline
\end{tabular}

VEGF: Vascular endothelial growth factor

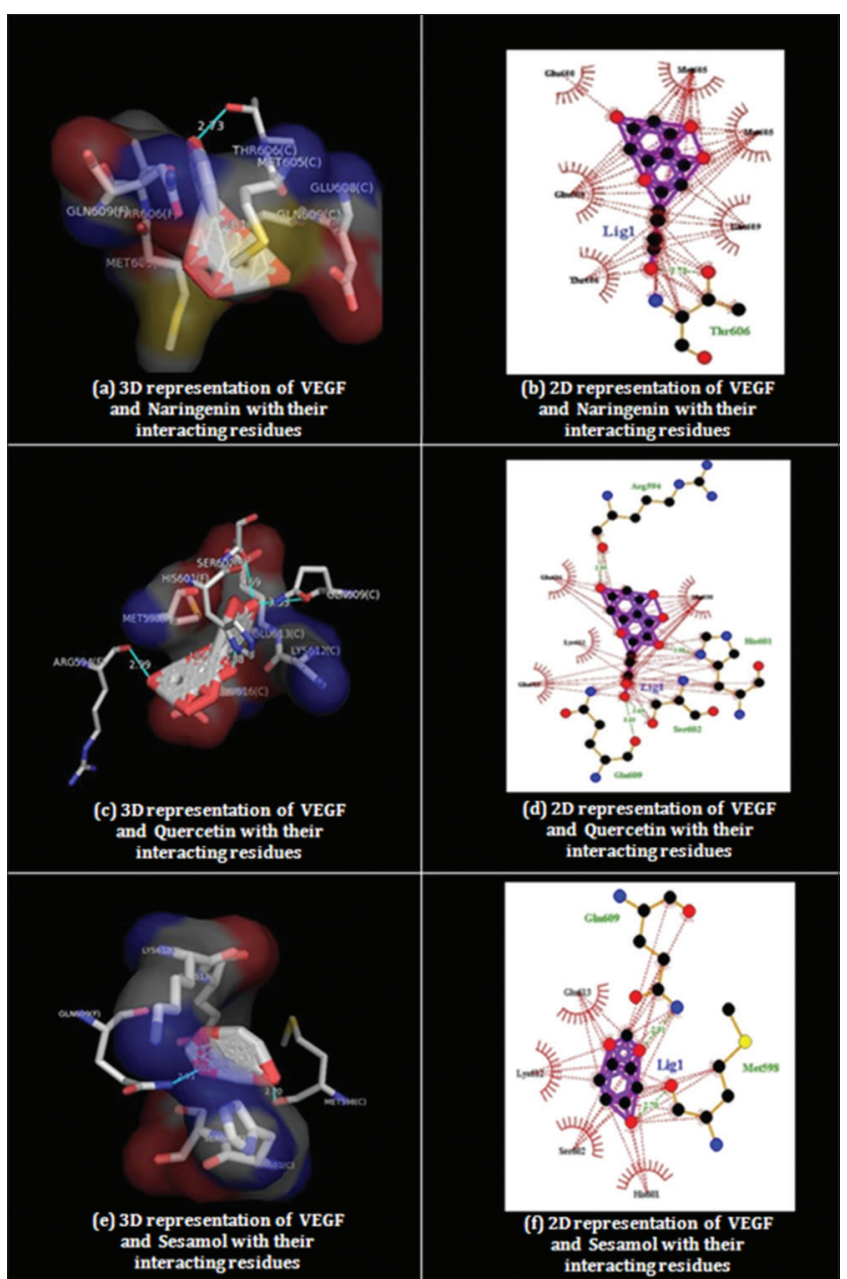

Fig. 4: Three-dimensional- and two-dimensional-representation of vascular endothelial growth factor and ligand interaction

\section{ACKNOWLEDGMENT}

The authors would like to thank senior management of Delhi Technological University for their encouragement and support. The authors would also like to thank Technical Education Quality Improvement Program-II for providing Fellowship to NKJ.

\section{REFERENCES}

1. Schlachetzki JC, Saliba SW, Oliveira AC. Studying neurodegenerative diseases in culture models. Rev Bras Psiquiatr 2013;35 Suppl 2:S92-100.

2. Hohman TJ, Bell SP, Jefferson AL; Alzheimer's Disease Neuroimaging Initiative. The role of vascular endothelial growth factor in neurodegeneration and cognitive decline: Exploring interactions with biomarkers of Alzheimer disease. JAMA Neurol 2015;72:520-9.

3. Storkebaum E, Carmeliet P. VEGF: A critical player in neurodegeneration. J Clin Invest 2004;113(1):14-8

4. Lange C, Storkebaum E, de Almodóvar CR, Dewerchin M, Carmeliet P. Vascular endothelial growth factor: A neurovascular target in neurological diseases. Nat Rev Neurol 2016;12(8):439-54.

5. Schneider SA, Dusek P, Hardy J, Westenberger A, Jankovic J, Bhatia KP. Genetics and pathophysiology of neurodegeneration with brain iron accumulation (NBIA). Curr Neuropharmacol 2013;11(1):59-79.

6. Wyss-Coray T, Rogers J. Inflammation in Alzheimer disease-a brief review of the basic science and clinical literature. Cold Spring Harb Perspect Med 2012;2(1):a006346.

7. Vagnucci AH Jr, Li WW. Alzheimer's disease and angiogenesis. Lancet 2003;361(9357):605-8

8. Dalton HJ, Armaiz-Pena GN, Gonzalez-Villasana V, Lopez-Berestein G, Bar-Eli M, Sood AK. Monocyte subpopulations in angiogenesis. Cancer Res 2014;74(5):1287-93.

9. Pandey KB, Rizvi SI. Plant polyphenols as dietary antioxidants in human health and disease. Oxid Med Cell Longev 2009;2(5):270-8

10. Raza SS, Khan MM, Ahmad A, Ashafaq M, Islam F, Wagner AP, et al. Neuroprotective effect of naringenin is mediated through suppression of NF-?B signaling pathway in experimental stroke. Neuroscience 2013;230:157-71.

11. Haleagrahara N, Siew CJ, Mitra NK, Kumari M. Neuroprotective effect of bioflavonoid quercetin in 6-hydroxydopamine-induced oxidative stress biomarkers in the rat striatum. Neurosci Lett 2011;500(2):139-43.

12. Sarkar A, Angeline MS, Anand K, Ambasta RK, Kumar P. Naringenin and quercetin reverse the effect of hypobaric hypoxia and elicit neuroprotective response in the murine model. Brain Res 2012;1481:59-70.

13. Kumar P, Kalonia H, Kumar A. Protective effect of sesamol against 3-nitropropionic acid-induced cognitive dysfunction and altered glutathione redox balance in rats. Basic Clin Pharmacol Toxicol 2010;107(1):577-82.

14. Maida E, Se BB, Divakar S, Geetha G. Ligand based pharmacophore modeling, virtual screening and molecular docking studies to design novel pancreatic lipase inhibitors. Int J Pharm Pharm Sci 2017;9(4):48-64.

15. Rigoberto VG, Rodolfo AV, Vera LP. Chemical compounds and biological activity of an extract from Bougainvillea $x$ buttiana (Var. Rose) holttum and standl. Int J Pharm Pharm Sci 2017;9(3):42-6.

16. Dharani RS, Ranjitha R, Sripathi R, Muhammad KS, Ravi S. Docking studies in target proteins involved in antibacterial action mechanisms: Alkaloids isolated from scutellariagenus. Asian J Pharm Clin Res 2016;9(5):121-5

17. Manjula J, Maheswari R. Biological and docking studies of novel aroylhydrazones. Int J Pharm Pharm Sci 2017;9(5):81-5. 